\section{OHP-054 LIFTING THE QUALITY OF THE DAILY SERVICE, BY OBTAINING CONSENSUS BETWEEN PHARMACY TECHNICIANS WITH REGARD TO GENERIC DRUG PRESCRIPTIONS}

\section{doi:10.1136/ejhpharm-2013-000276.428}

'S Beier, ${ }^{2} \mathrm{D}$ Thomsen, 'M Wellner. 'Region Hovedstadens Apotek, Medicinservice Herlev Hospital, Herlev, Denmark; ${ }^{2}$ Region Hovedstadens Apotek, Medicinservice Hillerød Hospital, Herlev, Denmark

Background After structural changes in the clinical pharmaceutical services at Herlev Hospital (DK), there are now two different pharmacy technicians serving the same ward. This structural change revealed considerable differences in the daily routines and service. This was unsatisfactory to the wards and had a negative effect on the working environment.

Purpose To reach consensus about the level of daily service by focusing on changing prescribing toward generic stock drugs and thereby enabling all staff to conduct a uniform level of service.

Materials and Methods A new educational programme was drawn up and implemented. The educational focus was on drugs that had been obtained by an EU tender, changing prescribing habits toward generic drugs in stock, within ATC-group C and N, and interprofessional communication. Support tools for the new practise were introduced.

The impact of the intervention was measured by an anonymous questionnaire answered by the pharmacy technicians at Herlev Hospital.

Results The response rate was $75 \%$.

The answers on the benefit of the new educational programme were:

No benefit at all: $0 \%$, minor benefit: $8 \%$, fair benefit: $53 \%$, high benefit: $22 \%$ and no answer: $17 \%$.

The answers on the benefit of the support tools were:

No benefit at all: $11 \%$, minor benefit: $24 \%$, fair benefit: $28 \%$, high benefit: $28 \%$ and no answer: $9 \%$.

All who answered the question (78\%) agreed that a consensus had been reached on the daily services. There were variations in answers about shifting prescribing towards generic drugs in stock after the intervention, some experienced a considerable effect and others experienced no difference.

Conclusions The intervention was shown to be effective. Consensus was reached on the level of daily service. The staff is now more comfortable with the daily routines, but some variation in the day to day work still remains.

No conflict of interest.

\section{OHP-055 MANAGEMENT OF HYPERTENSION IN TYPE 2 DIABETIC OUTPATIENTS}

doi:10.1136/ejhpharm-2013-000276.429

${ }^{1}$ S Khiter, ${ }^{2} \mathrm{E}$ Speyer, ${ }^{1} \mathrm{I}$ May, ${ }^{3} \mathrm{O}$ Ziegler, ${ }^{1} \mathrm{~A}$ Giesenfeld, ${ }^{3} \mathrm{P}$ Böhme. ${ }^{1} \mathrm{CHU}$ NANCY Hôpitaux de Brabois, Pharmacy, Vandoeuvre les Nancy, France; ${ }^{2} \mathrm{CHU}$ NANCY Hôpitaux de Brabois, Epidemiology and clinical evaluation, Vandoeuvre les Nancy, France; ${ }^{3} \mathrm{CHU}$ NANCY Hôpitaux de Brabois, Diabetology, Vandoeuvre les Nancy, France

Background The control of blood pressure is one of the main objectives in type 2 diabetes mellitus (T2D) management, as well as glycaemic control.

Purpose The first objective of this study was to describe the practise in hypertensive drug management in a cohort of DT2 patients from a diabetology department of a university hospital and to compare this practise with the current guidelines for hypertension treatment (HGs).
Materials and Methods This retrospective study examined T2D outpatients who came to the diabetology department between June and November 2010 for an annual cheque-up. Clinical and therapeutic data were extracted. Patients' blood pressure levels were measured by an automated procedure (Dynamap).

Results The analysis was carried out on 803 patients (age: $64.9 \pm 8.9 \mathrm{yrs} ; 38.6 \%$ women). The combination of T2D with confirmed hypertension was frequent $(82.9 \%)$ and higher than the national results $(80 \%)$. This situation was associated with cardiovascular and renal complications for $21 \%$ and $22.4 \%$ of the patients, respectively. The average systolic and diastolic blood pressures were 132.9 and $71.3 \mathrm{mmHg}$, respectively. Recommended objective for DT2 patients $(<130 / 80 \mathrm{mmHg}$ ) was reached for $44.6 \%$ of the patients. Mono, dual and triple therapies were in accordance with the HGs in $100 \%, 95 \%$ and $85 \%$ of the patients, respectively. The effect of these different combinations, illustrated by the median of the blood pressure levels, was better for monotherapies (128.5/70.3 $\mathrm{mmHg}$ ) than for dual and triple therapies (132.5/72 and 131/70.8 $\mathrm{mmHg}$, respectively). $19 \%$ of patients had to take at least 4 antihypertensive drugs and the median of their systolic and diastolic blood pressures were 135.5 and $71 \mathrm{mmHg}$, respectively.

Conclusions In DT2 patients, blood pressure control should be improved, with for example earlier detection of hypertension and/ or therapeutic reinforcement. However, antihypertensive drug management seems to be in accordance with the French official guidelines. The development of new drugs and patient education programmes may improve patient adherence.

No conflict of interest.

\section{OHP-056 MANAGEMENT OF THE VIAL RESIDUES IN AN INTRAVENOUS CHEMOTHERAPY UNIT OF A TERTIARY HOSPITAL}

doi:10.1136/ejhpharm-2013-000276.430

JE Megías Vericat, J Ruiz Ramos, J Reig Aguado, MJ Ausina Ausina, C Borrell García, MJ Esteban Mensua, E López Briz, JL Poveda Andrés. Hospital Universitario y Politécnico La Fe, Servicio de Farmacia, Valencia, Spain

Background Minimization of chemotherapy costs has become a rational goal in today's economic environment.

Purpose To assess the cost savings achieved by optimising vial residues during chemotherapy preparation.

Materials and Methods A longitudinal prospective study was conducted in the Intravenous Chemotherapy Unit of the Pharmacy Service between 15 January and 31 March of 2012. We selected the six drugs with more potential cost saving (bevacizumab, bortezomib, liposomal doxorubicin, panitumumab, rituximab and trastuzumab). Data were collected with the Oncofarm software: number of patients, number of preparations, theoretical and actual number of vials used. For economic estimates the retail price (RRP) was used. Results During the study period, 365 preparations were administered to 190 patients; these required the potential use of 716 vials, but actually 545 vials were used, saving $219,538 €$ (33\% of the cost without recycling excess vials).

Data analysis showed that $81 \%$ of the total savings were achieved with only 2 drugs: bevacizumab (50\%, 80 vials, $€ 110,556)$ and ritux$\operatorname{imab}(31 \%, 50$ vials, $€ 67,752)$. Their high frequency of use ( $66 \%$ of preparations and $68 \%$ of patients), high cost and greater variability of prescribed doses, justifies these results.

Theoretical average costs of the preparations analysed without managing the residues of partially-used containers were 1,790 (SD:476) €/preparation and 3,568 (SD:642) €/patient. After savings were made the averages were 499 (SD:253) €/preparation and 965 (SD:389) €/patient. Rituximab (€836/preparation, $€ 1,063 /$ patient), bevacizumab ( $€ 700 /$ preparation, $€ 1,602 /$ patient) and panitumumab (€625/preparation, $€ 1,111 /$ patient) were the drugs with greater savings. 
We estimated the difference between potential savings if the adjustment had been perfect and the actual saving obtained $(€ 21,135)$, possibly caused by the preparation process or expiry of some reconstituted vials

Conclusions Residues management is a common practise to improve the efficiency of the preparation process. Optimizing this process of updating medicines' stabilities, recording the opening date on the vial, checking expiries and storage conditions, achieved significant savings in the cost of treatments.

No conflict of interest.

\section{OHP-057 MEASURES FOR PALIVIZUMAB COST CONTAINMENT ANALYSIS}

doi:10.1136/ejhpharm-2013-000276.431

M Bullejos Molina, J Nazco Casariego, I Rodriguez Pedrosa, J Gonzalez Garcia, I Gonzalez Perera. Hospital Universitario de Canarias, Servicio de Farmacia, La Laguna, Spain

Background Prescription RSV (Respiratory Syncytial Virus) immunoprophylaxis with palivizumab involves high pharmaceutical costs associated with paediatric services. It is necessary to establish protocols aimed at reducing the cost associated with these treatments, adjusted to the best cost-effectiveness criteria.

Purpose To assess whether the prescriptions are consistent with indications of greater efficiency; to assess the impact of the revision of the criteria in the last vaccination campaign.

Materials and Methods We analysed the cost associated with the use of palivizumab in the last six years, the criteria for indication of prophylaxis, and the impact of the restrictions introduced last season. The number of doses that can be administered has been restricted: a limitation for the higher-risk months (Nov-Jan), and more cost-effective presentations $(100 \mathrm{mg})$ are to be used.

We extracted from our hospital system (SAP) the dispensed prescriptions of palivizumab from September 2006 to February 2012 (5 vaccination campaigns) analysing the number of patients treated, number of doses per child, vaccination period, consumption distribution among different presentations, indication criteria and associated cost.

Results An examination of the last 6 vaccination campaigns shows the impact of the measures taken. We obtained a $35 \%$ cost reduction $(€ 98,875.25)$ compared to the average in recent seasons, and a $28 \%$ decrease in the number of children treated. The priority of using 100 $\mathrm{mg}$ vials meant a $63 \%$ reduction in the use of $50 \mathrm{mg}$ vials, which are less cost-effective. The largest decrease $(10 \%)$ in prescriptions was in premature infants between 29 and 35 weeks gestation. No vaccinations were done in March.

Conclusions Establishing agreed more restrictive criteria used in the selection of patients to be treated, limiting the months in which the vaccine can be administered and the preferential use of $100 \mathrm{mg}$ vials has brought about a $35 \%$ reduction in the cost associated with this treatment $(€ 98,875.25)$ compared to previous campaigns.

No conflict of interest.

\section{OHP-058 NEW RESPONSIBILITIES FOR PHARMACY TECHNICIANS: THE SKILLS MATRIX, A PERFECT TOOL FOR CHANGE MANAGEMENT}

doi:10.1136/ejhpharm-2013-000276.432

E Barbier, L Halcewicz, S Esposito, A Rieutord, S Barbault-Foucher. Hôpital Antoine Béclère, Pharmacy, Clamart, France

Background Our teaching hospital has a level 3 maternity unit and a neonatal intensive care unit for 29 preterm infants. Over 3,000 bags of paediatric parenteral nutrition are prescribed annually. Their production is outsourced to another hospital. Until 2010, only pharmacy residents and pharmacists were in charge of this activity.

Purpose To design and implement a skills matrix to shift this activity towards the hospital pharmacy technicians.

Materials and Methods A multidisciplinary working group (a pharmacist, a chief technician, a pharmacy resident, two pharmacist technicians (PTs)) defined Standard Operating Procedures (SOPs) needed and skill levels according to our Process Map. They established a training programme and finally a Gantt chart.

Results Our matrix includes two levels: level 2 consists of the delivery of parenteral nutrition; level 1 also includes ordering and checking nutrition bags, the management of nonconforming products and monthly management.

Of the 11 pharmacy technicians, 100\% gained level 2 and 55\% level 1 between January and May 2011 as defined. The activity shift was fully completed after 6 months. SOPs were reviewed and approved entirely during 2011. Experience feedback meetings have been set on a regular basis with the clinical ward to maintain standards since June 2012.

Pharmacy technicians have expanded their skills and this has enabled us to save pharmacists' time (0.3 Full Time Equivalent). PTs were examined again in September 2012 in order to assess their skills and knowledge after one year, using interactive real-life exercises.

Conclusions The skills matrix is a simple and attractive management tool for identifying needs, assessing and developing individual skills. It provides not only a clear insight into individual skills but also into transversal competencies in a Pharmacy Department. It is particularly adapted to conducting change in a peaceful and positive manner and very important for annual individual assessment.

No conflict of interest.

\section{OHP-059 OPTIMIZATION OF HIGH-IMPACT MEDICINES IN PAEDIATRICS}

doi:10.1136/ejhpharm-2013-000276.433

R Tamayo Bermejo, C Gallego Fernández, J González Chávez, M Ruiz de Villegas, I Muñoz Castillo. HRU CARLOS HAYA, Pharmacy, Málaga, Spain

Background High economic impact medicines are used off-label in paediatric situations, using adult presentations for lack of a paediatric form.

Purpose To justify preparing individualised medicines for paediatric use according to individual need; adaptation to increase safety and reduce costs.

Materials and Methods Retrospective review of high-impact medicines used in individualised treatment in paediatrics. Duration of study: 4 years. The medicines were included if they had been needed (adalimumab 35 months, anakinra 73 months and pegfilgrastim 50 months).

Data collection sources: Computer application in the pharmacotechnical area, software of the outpatient dispensing and management system. Personnel times were collected according to the Catalogue of Products and Billing (2nd edition 2009) and costs according to the Analytical Accounting Service. As these were standard sterile formulas the time and cost of pharmaceutical personnel were considered (standard operating procedure of a new product and successive validations), nurse (production) and technician (material preparation, labelling and packaging.

We compared the cost of dispensing the complete pharmaceutical form with individualised costs through sterile repackaging

Variables studied: patients, different types of dosages, number of syringes made, number of syringes consumed and associated costs. For economic valuation the cost of the commercial presentation and the personnel involved in the making were considered.

Results The 3 medicines identified were repacked from the adult branded product formulations. 